\title{
3D pediatric cranial bone imaging using high-resolution MRI for visualizing cranial sutures: a pilot study
}

\author{
Kamlesh B. Patel, MD, MSc, ${ }^{1}$ Cihat Eldeniz, PhD, ${ }^{2}$ Gary B. Skolnick, BS, ${ }^{1}$ \\ Udayabhanu Jammalamadaka, PhD, ${ }^{2}$ Paul K. Commean, BEE, ${ }^{2}$ Manu S. Goyal, MD, ${ }^{2}$ \\ Matthew D. Smyth, MD, ${ }^{3}$ and Hongyu An, DSc ${ }^{2}$ \\ 'Division of Plastic and Reconstructive Surgery, ${ }^{2}$ Mallinckrodt Institute of Radiology, and ${ }^{3}$ Department of Neurosurgery, \\ Washington University in St. Louis, Missouri
}

OBJECTIVE There is an unmet need to perform imaging in young children and obtain CT-equivalent cranial bone images without subjecting the patients to radiation. In this study, the authors propose using a high-resolution fast low-angle shot golden-angle 3D stack-of-stars radial volumetric interpolated breath-hold examination (GA-VIBE) MRI sequence that is intrinsically robust to motion and has enhanced bone versus soft-tissue contrast.

METHODS Patients younger than 11 years of age, who underwent clinical head CT scanning for craniosynostosis or other cranial malformations, were eligible for the study. 3D reconstructed images created from the GA-VIBE MRI sequence and the gold-standard CT scan were randomized and presented to 3 blinded reviewers. For all image sets, each reviewer noted the presence or absence of the 6 primary cranial sutures and recorded on 5-point Likert scales whether they recommended a second scan be performed.

RESULTS Eleven patients (median age 1.8 years) underwent MRI after clinical head CT scanning was performed. Five of the 11 patients were sedated. Three clinicians reviewed the images, and there were no cases, either with CT scans or MR images, in which a reviewer agreed a repeat scan was required for diagnosis or surgical planning. The reviewers reported clear imaging of the regions of interest on $99 \%$ of the CT reviews and $96 \%$ of the MRI reviews. With CT as the standard, the sensitivity and specificity of the GA-VIBE MRI sequence to detect suture closure were $97 \%$ and $96 \%$, respectively ( $n=198$ sutures read).

CONCLUSIONS The 3D reconstructed images using the GA-VIBE sequence in comparison to the CT scans created clinically acceptable cranial images capable of detecting cranial sutures. Future directions include reducing the scan time, improving motion correction, and automating postprocessing for clinical utility.

https://thejns.org/doi/abs/10.3171/2020.4.PEDS20131

KEYWORDS craniosynostosis; MRI; imaging; skull; 3D; craniofacial

$\mathrm{D}$ IAGNOSTIC imaging involving CT scans obtained in patients with craniosynostosis has been questioned due to radiation; however, most surgical teams continue to obtain CT scans to confirm the diagnosis. ${ }^{1,2}$ Head CT scanning protocols for craniosynostosis vary by institution, with some groups obtaining up to 3 scans-before surgery, 1-3 days after the operation, and 1 year postoperatively. ${ }^{3}$ The preoperative CT scan is obtained to evaluate cranial dysmorphology and the patency of the cranial sutures. The postoperative head CT scans, on the other hand, are used to assess postoperative outcomes, to evaluate cranial defects, and to surgically plan secondary procedures. The CT scans are also used to as- sess intracranial abnormalities that may be coincidental or be associated with the condition (e.g., enlarged ventricles, excess fluid in subarachnoid spaces, arachnoid cyst, Chiari malformation/cerebellar herniation).

State-of-the-art head CT scanning provides high-resolution images that allow for sophisticated 3D reconstructions, with a scan duration of approximately 5 seconds. Despite the benefits of CT, concerns have been raised in the craniofacial community concerning the risks from radiation exposure. ${ }^{3-5}$ Recent efforts have been made to help minimize the radiation by utilizing low-dose protocols. ${ }^{6}$ Nonetheless, major organizations agree that even a very low-dose scan should not be considered safe?

ABBREVIATIONS GA = golden angle; ICC = intraclass correlation coefficient; PETRA = pointwise encoding time reduction with radial acquisition; VIBE = volumetric interpolated breath-hold examination. 
Ionizing radiation from $\mathrm{CT}$ scanning increases the risk of cancer, with children younger than 5 years of age at greatest risk. ${ }^{8-13}$ Patients with craniosynostosis often undergo repeated head CT scanning as part of clinical follow-up, exacerbating the cumulative risk. The National Cancer Institute reported, based on the findings by Pearce et al., ${ }^{11}$ that radiation exposure from multiple head CT scans triples the risk of leukemia and brain cancer in children. In addition, the use of sedation, if necessary, to reduce patient motion is associated with risks such as cardiopulmonary complications and neurological effects. ${ }^{14-16}$ Unfortunately, there is currently no clinically viable alternative to CT for imaging cranial bone that avoids both radiation and sedation.

MRI has the potential to be a safe alternative to CT because MRI does not expose patients to ionizing radiation. Eley et al. proposed a "black-bone" protocol that results in dark signal intensities in the osseous tissue, enabling extraction of the cranium after an inversion of the image intensities. ${ }^{17}$ However, black-bone methods are not widely utilized because of the following: 1) suboptimal osseous/ soft-tissue contrast, 2) motion artifacts in nonsedated patients, and 3) the need for manual, operator-dependent postprocessing. ${ }^{17,18}$ As a result, the capability of MRI to produce CT-like 3D reconstructed images of the cranium has been limited..$^{19}$ Rofsky et al. developed a 3D volumetric interpolated breath-hold examination (VIBE) method for rapid T1-weighted body imaging within a single breath hold within 20 seconds. ${ }^{20}$ A conventional VIBE scan acquires k-space data using cartesian encoding, and it relies on breath hold to minimize motion artifacts. As a result, the spatial resolution of VIBE is limited due to a short acquisition time, and motion artifacts may arise if patients have difficulty holding their breath. To overcome these issues, a radial VIBE method using a golden-angle acquisition scheme (GA-VIBE) was introduced for its intrinsic insensitivity to motion and motion correction capability for body imaging. ${ }^{21,22}$ An azimuthal angle of $111.25^{\circ}$, based on the golden ratio, is used to achieve nearly uniform radial k-space coverage during any duration of acquisition. In light of the strengths of the GA-VIBE demonstrated in body imaging, we adopted it for cranial bone imaging in place of the existing cartesian black bone MRI. In this study, we developed a GA-VIBE imaging protocol with the goal to improve osseous/soft-tissue contrast and reduce sensitivity to motion. Moreover, we sought to evaluate its feasibility in pediatric patients evaluated for craniosynostosis and other cranial malformations by using a blinded clinical evaluation. This study has the potential to provide the craniofacial community an alternative imaging method that avoids the risks of radiation exposure.

\section{Methods \\ Study Design and Participants}

Recruitment began following institutional review board approval. Informed consent was obtained from the parent(s) of all participants. Pediatric patients, younger than 11 years, who received clinical head CT scans for craniosynostosis or other cranial malformation were considered. The study design was cross-sectional. The par- ticipant intervention was the radial MRI sequence that took approximately 5 minutes, either added to an already scheduled clinical MRI protocol or performed as a standalone research scan. Exclusion criteria consisted of the following: 1) children who had a cranial procedure after CT scanning and before MRI, 2) MRI more than 1 year after the CT scanning, and 3) contraindications to MRI.

Demographic and scan information was collected from the electronic medical record. This included sex, diagnosis, age at the time of CT and MRI, use of sedation (yes/ no), and type of MR scanner. Although the main study outcome was the visibility of cranial sutures for the diagnosis of craniosynostosis, patients with and without craniosynostosis were considered. Initially, only the patients who were also scheduled for clinical MRI were considered. Later, to increase the number and proportion of participants scanned without sedation, recruits included patients who were asked to return for a separate researchonly, nonsedated MRI examination.

\section{Imaging and Processing}

CT scans followed standard clinical pediatric methods. A multislice SOMATOM Definition Flash or SOMATOM Force CT scanner (Siemens Medical Systems) was used, with slice thickness ranging from 0.6 to $1 \mathrm{~mm}$ and pixel spacing ranging from $0.31 \times 0.31 \mathrm{~mm}$ to $0.39 \times 0.39 \mathrm{~mm}$ and an approximate scan time of $5.3 \pm 2.1$ seconds (range 3.5-10.5 seconds). The following CT parameters were applied to acquire the images: 0.5 -second rotation time, 64 $\times 0.6-\mathrm{mm}$ collimation, $220 \mathrm{mAs}, 70-150 \mathrm{kVp}$, a pitch of one, and a $512 \times 512$ matrix.

MR images were acquired using a 3T Prisma, a 3T VIDA, or a 3T Biograph mMR scanner (Siemens Healthineers). A fast low-angle shot golden-angle 3D stack-ofstars radial VIBE sequence (GA-VIBE) ${ }^{23}$ was used to acquire images with the following imaging parameters: TR/ $\mathrm{TE}=4.84 \mathrm{msec} / 2.47 \mathrm{msec}$, field of view $192 \mathrm{~mm}$, bandwidth $410 \mathrm{~Hz} /$ pixel, 224 slices per slab, transverse orientation, flip angle $3^{\circ}$, acquisition matrix $320 \times 320$, voxel size $0.6 \times 0.6 \times 0.8 \mathrm{~mm}$, and total number of radial lines 400 , for a scan duration of 5 minutes, 4 seconds. Given that several types of tissues exist in the head, the GA-VIBE protocol is designed to maximize the image contrast between bone and all nonosseous tissues. More specifically, an echo time value was chosen to keep the water and fat signals in phase, and a small flip angle $\left(3^{\circ}\right)$ was used to introduce proton-density weighting to preserve a high signal in the soft tissue, CSF, and adipose tissue. This led to the osseous tissues having a very low signal due to their low proton density and rapid signal decay (i.e., high R2*).

Following scanning, CT and MR images were manually processed in 3D Slicer, and 3D reconstructions of both the CT and MR images were obtained. ${ }^{24} \mathrm{MR}$ images were registered to $\mathrm{CT}$ scans for each participant using a rigid-body plus scaling transformation. MR images were then processed with bias correction (using the N4iTK algorithm), masking, intensity inversion, recursive Gaussian filtering and volume rendering to yield 3D images of the cranium. The MRI processing time ranged from approximately 30 minutes to 2 hours per patient. CT scan rendering follows standard clinical pediatric methods and 

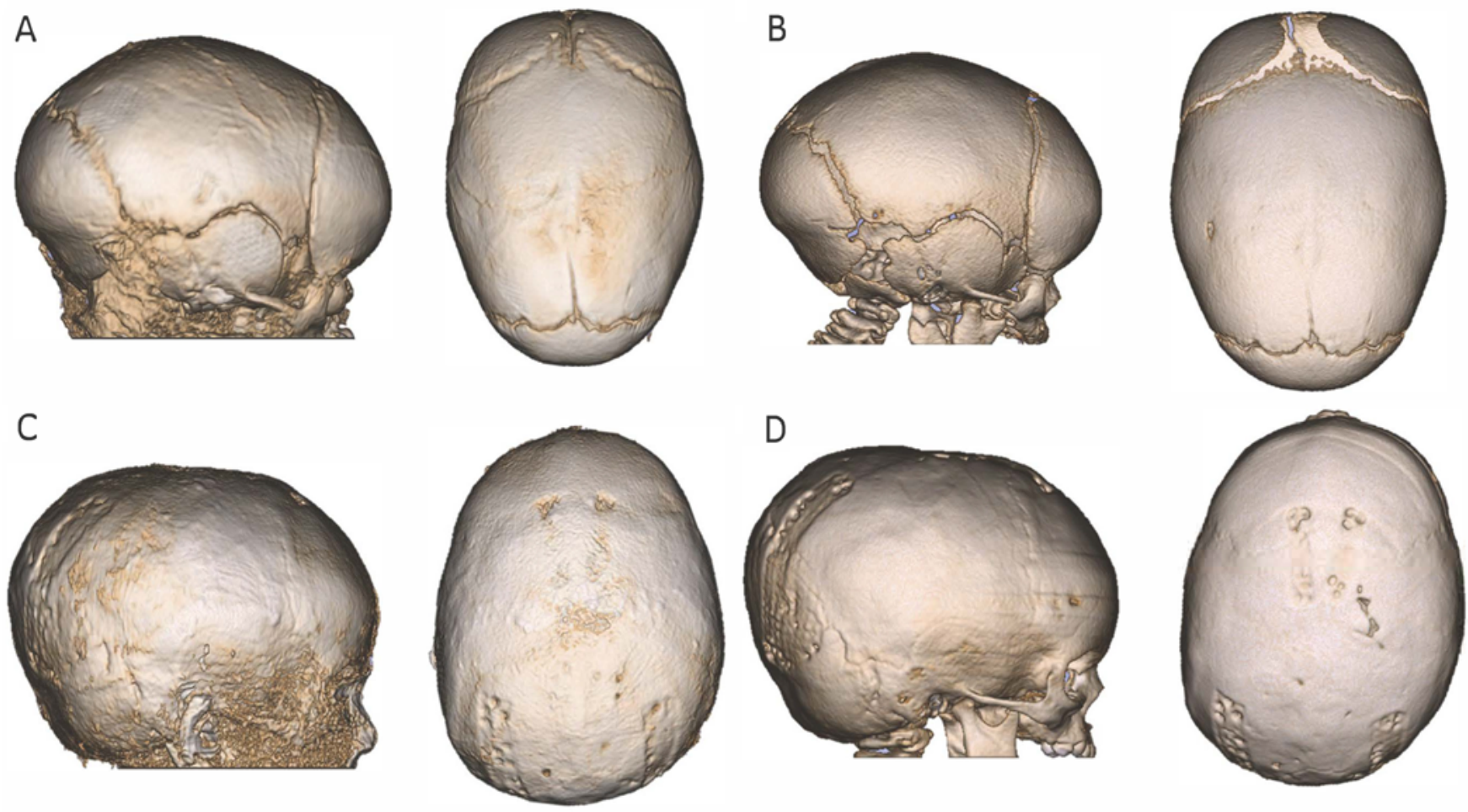

FIG. 1. Sample profile and vertex views of MR images and CT scans from four separate slide decks. MR image (A) and CT scan (B) of patient 4, a 6-week-old boy with sagittal synostosis, and MR image (C) and CT scan (D) of patient 9, an 18-month-old boy with sagittal synostosis 1 year postoperatively. Figure is available in color online only.

employs the preset bone intensity threshold. The CT processing time was approximately 10 minutes per patient. Rendered images of the reconstructions in different views were then captured at $15^{\circ}$ increments in horizontal and vertical rotations for a total of 48 images (24 horizontal and 24 vertical) per patient scan. The screenshots for the pair of vertically and horizontally rotated MR images were later compiled into a slide deck for review with a total of 24 slides, one for each rotation angle. The screenshots for the CT scans were compiled in the same way. Figure 1 shows sample slides from 2 patients.

\section{Clinical Evaluation}

Three raters, a craniofacial plastic surgeon (K.B.P.), pediatric neurosurgeon (M.D.S.), and neuroradiologist (M.S.G.), compared the 3D reconstructed images from the MRI data to the gold-standard CT-based images. The assessment was based on 48 3D reconstructed cranial images. The images were shown to each reviewer as two side-by-side 3D reconstructions that were vertically and horizontally rotated in $15^{\circ}$ increments, as shown in Fig. 1. The reviewers were blinded to scan modality (MRI or CT) and patient information. They independently assessed the anatomical presence or absence (open, partially open, or closed) of 6 cranial sutures on both the CT and MR 3D images for each participant: sagittal, metopic, right and left coronal, and right and left lambdoid. The reviewers were also asked to rate the visibility of the sutures, that is, the clarity of the images on the regions of interest, using a 5 -point Likert scale. If there was disagreement on suture closure among the reviewers after the evaluation of the CT and MR images, a second round of reviews was performed to confirm the disagreement or correct mistaken responses.

In addition, for each set of images, the reviewers also noted on 5-point Likert scales if the scan quality was acceptable for diagnosis and for presurgical planning, and whether or not they would recommend a second scan be performed to allow for clinical diagnosis or presurgical planning. In the analysis of responses, the top 2 points in the Likert scale were consolidated; for example, a scan was considered acceptable if a reviewer either "agreed" or "strongly agreed" that the scan was acceptable. Responses were recorded in a REDCap database developed for this study.

Block randomization was used to shuffle the order in which CT and MR imaging data were presented to the reviewers. One MRI slide deck and one CT slide deck were selected at random and resent to the reviewers for repeat assessment in order to determine intrarater reliability.

\section{Statistical Analysis}

Descriptive statistics on patient demographics and scan information were calculated. Frequencies of reviewer responses to all questions were obtained. The Mann-Whitney U-test was used to compare reviews of MR images obtained in sedated and nonsedated patients. Sensitivity and specificity of the MR-based images to detect suture fusion were calculated using CT as the standard of reference. (There were cases of reviewer disagreement on 
TABLE 1. Patient demographics and imaging data

\begin{tabular}{rrrrrl}
\hline $\begin{array}{c}\text { Patient } \\
\text { No. }\end{array}$ & Sex & $\begin{array}{c}\text { Age at } \\
\text { MRI } \\
\text { (yrs) }\end{array}$ & $\begin{array}{c}\text { No. of } \\
\text { Days } \\
\text { After CT }\end{array}$ & $\begin{array}{c}\text { Sedated } \\
\text { for MRI }\end{array}$ & \multicolumn{1}{c}{ Diagnosis } \\
\hline 1 & M & 4.8 & 190 & Yes & Arachnoid cyst \\
\hline 2 & M & 0.8 & 5 & Yes & Encephalocele \\
\hline 3 & M & 1.4 & 101 & No & Hydrocephalus \\
\hline 4 & M & 0.1 & 9 & No & Sagittal synostosis \\
\hline 5 & M & 1.6 & 12 & No & $\begin{array}{c}\text { Sagittal \& bi-lambdoid } \\
\text { synostosis }\end{array}$ \\
\hline 6 & M & 1.8 & 12 & Yes & Sagittal synostosis \\
\hline 7 & F & 2.7 & 193 & Yes & Metopic synostosis \\
\hline 8 & F & 9.2 & 47 & Yes & Frontonasal dysplasia \\
\hline 9 & M & 1.5 & 56 & No & Sagittal synostosis \\
\hline 10 & M & 7.6 & 14 & No & Crouzon syndrome \\
\hline 11 & F & 8.9 & 0 & No & Crouzon syndrome \\
\hline
\end{tabular}

suture closure based on CT scanning. Each reviewer's individual response was used as the reference for the corresponding MR images.) Intrarater reliability was assessed with two-way random single-measures intraclass correlation coefficients (ICCs). Interrater reliability was assessed using two-way mixed-effects single-measures ICC. Fisher's exact test was performed using $\mathrm{R}$ version 3.3.2. ${ }^{25}$ All other analyses were performed using SPSS version 26 (IBM Corp.). Significance was set a priori at 0.05 .

\section{Results}

\section{Participant Profiles}

In total, 14 participants were recruited. The GA-VIBE sequence was used for 12 participants between January 2019 and January 2020. MR images were not obtained for the 2 other candidates, a 16-month-old child and a 3-yearold child, due to patient noncompliance. Image acquisition on one of the 12 scans was inadequate for reconstruction due to severe motion and early termination of the scan, leaving 11 data sets for evaluation. Age at MRI in these 11 participants ranged from 3 weeks to 9 years (median age 1.8 years). The median time after CT scanning was 14 days (range 5-193 days). None of the patients were sedated for CT, whereas 5 of the $11 \mathrm{MR}$ images were acquired in patients who were sedated, per clinical routine. Seven of the patients were diagnosed with craniosynostosis (2 with Crouzon syndrome). The remaining 4 had other craniofacial malformations (Table 1).

\section{Imaging Quality and Acceptability}

Sixty-six reviews were returned for analysis (11 patients $\times 2$ imaging methods $\times 3$ reviewers). For each imaging method (CT and MRI), there were 198 assessments of suture closure and imaging quality in the regions of interest (11 patients $\times 6$ sutures $\times 3$ reviewers). Reviewers reported clear imaging of the regions of interest on 99\% of CT reviews (194/196 independent assessments, 2 missing) and 96\% of MRI reviews (189/196, 2 missing). A
TABLE 2. Sensitivity and specificity of MRI for cranial sutures

\begin{tabular}{lccc}
\hline & \multicolumn{3}{c}{ CT Finding } \\
\cline { 2 - 4 } MRI Finding & Open & Closed & Partially Closed \\
\hline Open & 96 & 0 & 3 \\
\hline Closed & 2 & 72 & 4 \\
\hline Partially closed & 2 & 3 & 14 \\
\hline
\end{tabular}

single reviewer deemed visibility of two regions of interest on a single MR image "not clear." The remaining reviews reported that the region of interest was "partially visible." Figure 1 depicts sample images. (Images from all scans are available in the Supplemental Data.)

In 65 of the 66 cases, the reviewer agreed that both MR images and CT scans were acceptable for diagnosis and surgical planning. In one instance (patient 5), the reviewer agreed the CT scan was acceptable for presurgical planning but was neutral toward the acceptability of the corresponding MR image. The distributions of Likert scale responses to the MR images of sedated and nonsedated patients did not differ significantly (Mann-Whitney U-test 432.5; $\left.\mathrm{N}_{\text {sedated }}=30, \mathrm{~N}_{\text {unsedated }}=36 ; \mathrm{p}=0.074\right)$. There were no cases, either with CT scans or MR images, in which a reviewer agreed a repeat scan was required for diagnosis or surgical planning.

\section{Survey Validity}

The validity of survey responses was assessed by examining the agreement between reviewer reports that 1) a scan was acceptable for diagnosis or planning and 2) there was no need for a repeat scan. There was an exact match (across the 5-point Likert scale) on 53 of 54 reviews (98\%) of CT reconstructions and on 52 of 54 reviews (96\%) of MRI reconstructions for diagnosis and for presurgical planning.

\section{Sensitivity and Specificity Regarding Suture Closure}

The sensitivity to detect suture closure from MR images was $97 \%$. Specifically, 93 of 96 determinations of closed or partially closed sutures (per CT scan) were also identified as closed or partially closed on MRI reviews. The specificity was 96\%: 96 MRI scan reviews of the 100 CT open suture reviews also found the sutures to be open (Table 2). Subanalysis restricted to nonsedated patients revealed similar sensitivity (96\%) and specificity (96\%).

\section{Response Reliability}

Interrater reliability on assessment of suture fusion was based on 108 observations. The agreement between reviewers was moderate for MR images (ICC $0.689 ; 95 \%$ CI $0.563-0.792$ ) and good for CT (ICC 0.768 ; 95\% CI $0.666-$ 0.849). ${ }^{26}$ More specifically, on review of CT reconstructions, reviewers agreed on suture closure in 48 of 54 cases (33 open, 13 partially closed, and 2 fully closed). There was disagreement in the assessment of the $\mathrm{CT}$ reconstructions on the closure of the remaining 6 sutures ( 1 metopic, 1 coronal, 2 lambdoid, and 2 sagittal). As described in the Methods section, for sensitivity and specificity analysis 
of suture closure, MRI responses were matched to CT responses by the reviewer.

All 3 reviewers also completed a duplicate MRI survey (for patient 2) and a duplicate CT survey (for patient 6). As there are 16 questions in each survey, this yielded a total of 96 observations to assess intrarater reliability. Intrarater reliability was found to be excellent with respect to scan quality (ICC $0.989 ; 95 \%$ CI $0.975-0.989$ ) and with respect to cranial suture visibility (ICC $1.000 ; 95 \%$ CI $1.000-1.000$ ) and suture presence or absence (ICC 1.000; 95\% CI 1.000-1.000).

\section{Discussion}

Head CT scanning is the most commonly performed pediatric CT modality. ${ }^{8,27}$ Its use in children has increased rapidly to around 2.2 million scans per year in the United States. ${ }^{8}$ The projected number of future radiation-induced cancers from head CT scans has been estimated to be 1210 per year (range 630-2370), and a case of leukemia is projected to result from 1 in every 5250 head scans performed in children younger than 5 years of age. ${ }^{8}$ Many children undergo repeated head CT scanning as part of clinical follow-up, exacerbating the cumulative risk. A focus for physicians treating patients with craniofacial anomalies has been to decrease the number of CT scans and implementing low-dose protocols. The ideal solution is to have a safe imaging method for bone that does not require radiation.

In our pilot study of 11 patients, we showed the potential value of the high-resolution GA-VIBE MRI sequence in obtaining images of the cranium. An ideal protocol for MR bone imaging maximizes the image contrast between bone and all other tissues. Accordingly, our GA-VIBE protocol was devised to preserve the signal in the soft tissues, the CSF, and the adipose tissue, while minimizing the signal from osseous tissues. We demonstrated promising results for this MR bone imaging approach in the diagnosis and surgical planning in pediatric patients across a wide age range (from 3 weeks to 9 years of age). The GA-VIBE sequence is a proprietary sequence from Siemens. For Siemens scanners, the product sequence is Star VIBE. GAVIBE is a special case of Star VIBE. Philips has a similar implementation called 3D VANE XD. To the best of our knowledge, we are not aware of a GE product sequence that is equivalent to Siemens Star VIBE. Since GE's LAVA sequence is equivalent to the Siemens VIBE and GE has a radial acquisition product sequence PROPELLER, a GAVIBE equivalent sequence can be implemented by combing LAVA and PROPELLER for GE scanners.

Eley et al. developed a black-bone protocol to differentiate bone from surrounding structures using a highresolution conventional 3D gradient echo sequence. ${ }^{17}$ The black-bone MRI methods took advantage of the vanishing bone signal that appears white after intensity inversion. Understanding the importance of 3D reconstructed cranial images for diagnosis and surgical planning, Eley et al. evaluated the feasibility of the black-bone sequence in producing 3D reconstructions of the cranium..$^{28}$ Their study involved 12 subjects, with all patients younger than 8 years of age and undergoing sedation for the MRI. They found the postprocessing of images to be time intensive but were able to produce 3D reconstructed images. Dremmen et al. applied the black-bone MRI sequence in pediatric patients with cranial fractures and found poor accuracy in the detection of linear fractures in comparison with the gold-standard CT scanning, and they reported a sensitivity of $67 \%$ and a specificity of $88 \% .{ }^{29}$ The black-bone MRI methods use a standard gradient-echo sequence with cartesian k-space sampling. As a result, they are very sensitive to motion artifacts.

More recently, an ultrashort echo time sequence using pointwise encoding time reduction with radial acquisition (PETRA; Siemens) was implemented to detect skull fractures in pediatric patients with a spatial resolution of 1 $\mathrm{mm}^{3} \cdot{ }^{30}$ Compared to a previous study using a conventional gradient-echo sequence, ${ }^{29}$ the PETRA scan improved the detection sensitivity (83\%) and specificity (100\%); however, the sensitivity of this method decreased to $50 \%$ in patients who did not receive sedation. In PETRA, though the outer k-space is filled with half radial spokes, the central portion of the k-space is still sampled with a cartesian trajectory. Therefore, similar to the black-bone cartesian scan, PETRA imaging remains significantly vulnerable to motion artifacts. Since movement is very common in pediatric patients, sedation has been routinely used to minimize motion artifacts in MRI. Unfortunately, sedation is associated with risks, including cardiopulmonary complications and neurological effects. Therefore, it is highly desirable to develop an MRI method that is less sensitive to motion in order to produce artifact-free 3D cranial bone images without the need for sedation. CT scanning takes only several seconds (approximately 5 seconds for a head CT scan), allowing CT to be performed without sedation in most children. A retrospective review reported that the sedation rate in head CT was $18 \%$ in patients younger than 5 years of age and $1 \%$ in children older than 5 years of age. ${ }^{31}$ However, for pediatric head MRI, the need for sedation is much higher due to the longer imaging time. The sedation rate in head MRI was approximately $60 \%-65 \%$ in patients who are 1-6 years of age and 32\% for all patients younger than 18 years of age..$^{32}$ Recently, efforts have been made to reduce the need for sedation when performing pediatric MRI by reducing the imaging time and by using a multidisciplinary team to coordinate imaging with the patient's biorhythms. ${ }^{33-35}$ However, in a significant proportion of cases, sedation is still required to obtain clinically acceptable images. Therefore, the goal is to develop a fast MRI sequence that can produce high-quality, artifact-free images.

Motion usually produces ghosting artifacts in cartesian k-space sampling in the conventional black-bone and PETRA methods, negatively impacting the image quality. In contrast, the GA-VIBE is intrinsically more robust to motion, owing to its radial $\mathrm{k}$-space sampling. We compared the motion robustness of the GA-VIBE scan and the black-bone scan in a normal pediatric participant (Fig. 2). This participant moved slightly during both scans. This slight motion caused ghosting artifacts in the black-bone scan (Fig. 2A and B), while the GA-VIBE scan had almost no artifacts (Fig. 2C and D). As a result, cranial sutures were visible on the $3 \mathrm{D}$ reconstructed cranial bone images 

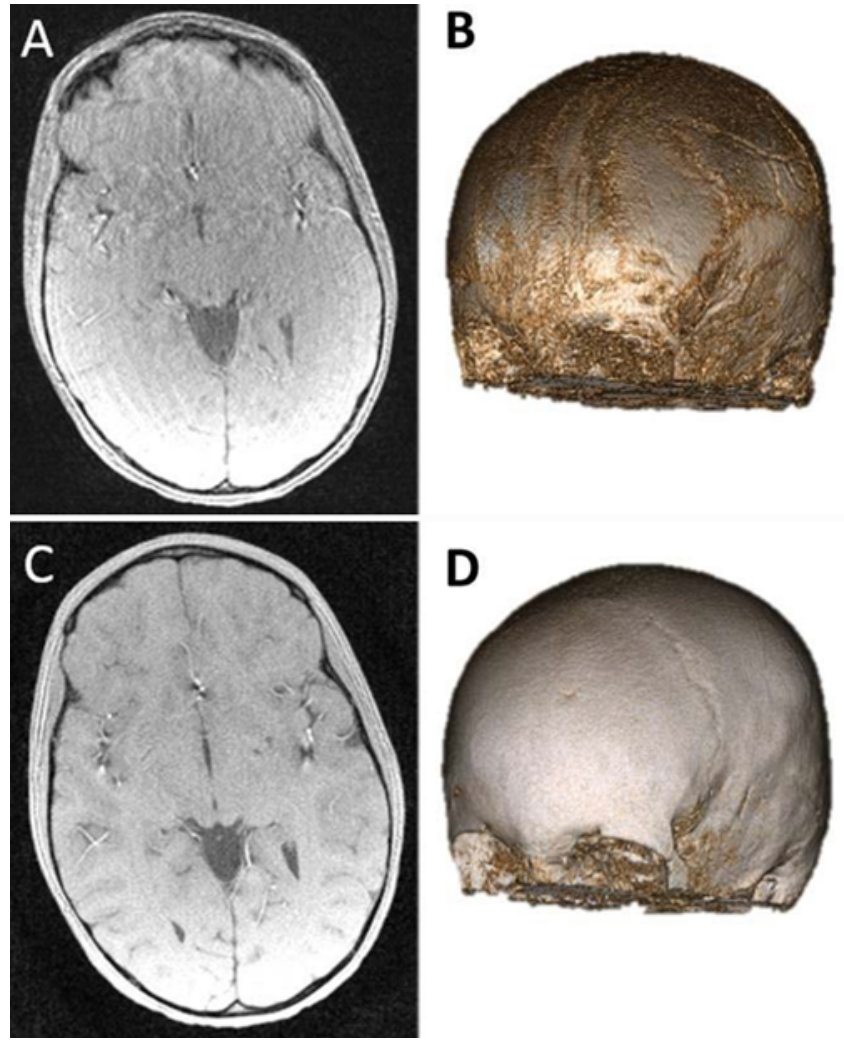

FIG. 2. MR images and the corresponding cranial bone reconstructions using the black-bone ( $A$ and $B$ ) and GA-VIBE (C and $D)$ scans obtained in a 6 -year-old nonsedated patient. Figure is available in color online only.

using the GA-VIBE scan, while they could not be distinguished in the motion-compromised black-bone scan. The robustness of GA-VIBE to small motions is further confirmed by the excellent sensitivity (96\%) and specificity (96\%) in detecting suture closure in our subgroup analysis in nonsedated patients $(\mathrm{n}=6)$. While Fig. 2 has demonstrated that the GA-VIBE sequence is insensitive to small motions, large degrees of motion can still degrade image quality in a GA-VIBE scan. In the future, we plan to develop a motion-correction method to further improve image quality in the presence of large degrees of motion..$^{22,36,37}$

The requirement to manually determine the signal threshold in separating bone from other tissues is another barrier to the MRI-based reconstruction of the cranial bone. Due to the poor signal contrast between bone and the surrounding tissues on MR images, the development of an operator-independent postprocessing method, using a traditional signal intensity approach, is challenging and precludes its clinical implementation. However, emerging advanced image-processing methods, such as deep learning methods, have the potential to generate CT-like images from MR images in a fully automated method. ${ }^{38,39}$

We were unable to perform complete MRI sequences in 3 patients due to poor compliance. Young children from 1 to 5 years of age are the most difficult to image without sedation. Keys to performing MRI without sedation involve using a dedicated child life team, scheduling during the child's naptime, reducing noise during the image acquisi- tion, developing shorter MRI sequences, and using foam cushions and vacuum immobilizers. ${ }^{40}$ Dean et al. reported an overall success rate of $97 \%$ in performing MRI in children younger than 4 years of age after utilization of these measures. ${ }^{41}$ Our group has also reported a high rate of acquiring clinical MRI scans for hydrocephalus evaluation. ${ }^{42}$ However, these methods are not available or IRB approved for a research MRI scan, resulting in a decreased rate of completion of scanning in nonsedated children.

\section{Conclusions}

In summary, GA-VIBE is a promising MRI method to achieve CT-like 3D cranial bone images in pediatric patients. The availability of the GA-VIBE sequence on both Siemens and Philips scanners and an equivalent sequence on GE scanners will allow for clinical translation. To allow for clinical utility, future directions will be as follows: 1) to improve MR image quality through motion correction to avoid sedation; 2) to automatically generate CT-like images using MR images acquired with deep learning methods; and 3) to demonstrate that 3D reconstructed high-resolution MR images can provide a viable alternative to CT cranial images in a large cohort of patients.

\section{Acknowledgments}

This research was supported by the Washington University Institute of Clinical and Translational Sciences grant no. UL1 TR000448 and the Mallinckrodt Institute of Radiology.

\section{References}

1. Doumit GD, Papay FA, Moores N, Zins JE. Management of sagittal synostosis: a solution to equipoise. J Craniofac Surg. 2014;25(4):1260-1265.

2. Kestle JRW, Lee A, Anderson RCE, et al. Variation in the management of isolated craniosynostosis: a survey of the Synostosis Research Group. J Neurosurg Pediatr. 2018;22(6):627-631.

3. Domeshek LF, Mukundan S Jr, Yoshizumi T, Marcus JR. Increasing concern regarding computed tomography irradiation in craniofacial surgery. Plast Reconstr Surg. 2009;123(4):1313-1320.

4. Fearon JA, Singh DJ, Beals SP, Yu JC. The diagnosis and treatment of single-sutural synostoses: are computed tomographic scans necessary? Plast Reconstr Surg. 2007;120(5):1327-1331.

5. Binning M, Ragel B, Brockmeyer DL, et al. Evaluation of the necessity of postoperative imaging after craniosynostosis surgery. J Neurosurg. 2007;107(1)(suppl):43-45.

6. Stewart NM, Hallac RR, Chou PY, et al. Craniofacial flash: minimizing radiation dose in pediatric craniofacial computed tomography. J Craniofac Surg. 2018;29(7):1751-1754.

7. Radiation risks and pediatric computed tomography (CT): a guide for health care providers. National Cancer Institute. September 4, 2018. Accessed April 23, 2020. https://www. cancer.gov/about-cancer/causes-prevention/risk/radiation/ pediatric-ct-scans

8. Miglioretti DL, Johnson E, Williams A, et al. The use of computed tomography in pediatrics and the associated radiation exposure and estimated cancer risk. JAMA Pediatr. 2013;167(8):700-707.

9. Brenner DJ, Elliston CD, Hall EJ, Berdon WE. Estimates of the cancer risks from pediatric CT radiation are not merely theoretical: comment on "Point/counterpoint: in x-ray com- 
puted tomography, technique factors should be selected appropriate to patient size. Against the proposition." Med Phys. 2001;28(11):2387-2388.

10. Parker L. Computed tomography scanning in children: radiation risks. Pediatr Hematol Oncol. 2001;18(5):307-308.

11. Pearce MS, Salotti JA, Little MP, et al. Radiation exposure from CT scans in childhood and subsequent risk of leukaemia and brain tumours: a retrospective cohort study. Lancet. 2012;380(9840):499-505.

12. Smyth MD, Narayan P, Tubbs RS, et al. Cumulative diagnostic radiation exposure in children with ventriculoperitoneal shunts: a review. Childs Nerv Syst. 2008;24(4):493-497.

13. Brenner DJ, Hall EJ. Computed tomography-an increasing source of radiation exposure. $N$ Engl J Med. 2007;357(22):2277-2284.

14. Coté CJ, Karl HW, Notterman DA, et al. Adverse sedation events in pediatrics: analysis of medications used for sedation. Pediatrics. 2000;106(4):633-644.

15. Kannikeswaran N, Mahajan PV, Sethuraman U, et al. Sedation medication received and adverse events related to sedation for brain MRI in children with and without developmental disabilities. Paediatr Anaesth. 2009;19(3):250-256.

16. Malviya S, Voepel-Lewis T, Eldevik OP, et al. Sedation and general anaesthesia in children undergoing MRI and CT: adverse events and outcomes. Br J Anaesth. 2000;84(6):743-748.

17. Eley KA, McIntyre AG, Watt-Smith SR, Golding SJ. "Black bone" MRI: a partial flip angle technique for radiation reduction in craniofacial imaging. Br J Radiol. 2012;85(1011):272-278.

18. Eley KA, Watt-Smith SR, Golding SJ. "Black bone" MRI: a potential alternative to $\mathrm{CT}$ when imaging the head and neck: report of eight clinical cases and review of the Oxford experience. Br J Radiol. 2012;85(1019):1457-1464.

19. Eley KA, Watt-Smith SR, Golding SJ. Three-dimensional reconstruction of the craniofacial skeleton with gradient echo magnetic resonance imaging ("black bone"): what is currently possible? J Craniofac Surg. 2017;28(2):463-467.

20. Rofsky NM, Lee VS, Laub G, et al. Abdominal MR imaging with a volumetric interpolated breath-hold examination. $R a$ diology. 1999;212(3):876-884.

21. Winkelmann S, Schaeffter T, Koehler T, et al. An optimal radial profile order based on the Golden Ratio for time-resolved MRI. IEEE Trans Med Imaging. 2007;26(1):68-76.

22. Grimm R, Fürst S, Souvatzoglou M, et al. Self-gated MRI motion modeling for respiratory motion compensation in integrated PET/MRI. Med Image Anal. 2015;19(1):110-120.

23. Peters DC, Korosec FR, Grist TM, et al. Undersampled projection reconstruction applied to MR angiography. Magn Reson Med. 2000;43(1):91-101.

24. Fedorov A, Beichel R, Kalpathy-Cramer J, et al. 3D Slicer as an image computing platform for the Quantitative Imaging Network. Magn Reson Imaging. 2012;30(9):1323-1341.

25. $R$ : A language and environment for statistical computing. Version 3.3.2. R Foundation for Statistical Computing; 2019.

26. Koo TK, Li MY. A guideline of selecting and reporting intraclass correlation coefficients for reliability research. $J$ Chiropr Med. 2016;15(2):155-163.

27. Dorfman AL, Fazel R, Einstein AJ, et al. Use of medical imaging procedures with ionizing radiation in children: a population-based study. Arch Pediatr Adolesc Med. 2011;165(5):458-464.

28. Eley KA, Watt-Smith SR, Sheerin F, Golding SJ. "Black Bone" MRI: a potential alternative to $\mathrm{CT}$ with three-dimensional reconstruction of the craniofacial skeleton in the diagnosis of craniosynostosis. Eur Radiol. 2014;24(10):2417-2426.

29. Dremmen MHG, Wagner MW, Bosemani T, et al. Does the addition of a "black bone" sequence to a fast multisequence trauma MR protocol allow MRI to replace CT after traumatic brain injury in children? AJNR Am J Neuroradiol. 2017;38(11):2187-2192.
30. Kralik SF, Supakul N, Wu IC, et al. Black bone MRI with $3 \mathrm{D}$ reconstruction for the detection of skull fractures in children with suspected abusive head trauma. Neuroradiology. 2019;61(1):81-87.

31. Goldwasser T, Bressan S, Oakley E, et al. Use of sedation in children receiving computed tomography after head injuries. Eur J Emerg Med. 2015;22(6):413-418.

32. Uffman JC, Tumin D, Raman V, et al. MRI utilization and the associated use of sedation and anesthesia in a pediatric ACO. J Am Coll Radiol. 2017;14(7):924-930.

33. Dong SZ, Zhu M, Bulas D. Techniques for minimizing sedation in pediatric MRI. J Magn Reson Imaging. 2019;50(4):1047-1054.

34. Lindberg DM, Stence NV, Grubenhoff JA, et al. Feasibility and accuracy of fast MRI versus CT for traumatic brain injury in young children. Pediatrics. 2019;144(4):e20190419.

35. Isaacs AM, Shimony JS, Morales DM, et al. Feasibility of fast brain diffusion MRI to quantify white matter injury in pediatric hydrocephalus. $J$ Neurosurg Pediatr. 2019;24(4):461-468.

36. Lin W, Guo J, Rosen MA, Song HK. Respiratory motioncompensated radial dynamic contrast-enhanced (DCE)MRI of chest and abdominal lesions. Magn Reson Med. 2008;60(5):1135-1146.

37. Paul J, Divkovic E, Wundrak S, et al. High-resolution respiratory self-gated golden angle cardiac MRI: comparison of self-gating methods in combination with k-t SPARSE SENSE. Magn Reson Med. 2015;73(1):292-298.

38. Han X. MR-based synthetic CT generation using a deep convolutional neural network method. Med Phys. 2017;44(4):1408-1419.

39. Gong K, Yang J, Kim K, et al. Attenuation correction for brain PET imaging using deep neural network based on Dixon and ZTE MR images. Phys Med Biol. 2018;63(12):125011.

40. Barkovich MJ, Xu D, Desikan RS, et al. Pediatric neuro MRI: tricks to minimize sedation. Pediatr Radiol. 2018;48(1):50-55.

41. Dean DC III, Dirks H, O'Muircheartaigh J, et al. Pediatric neuroimaging using magnetic resonance imaging during nonsedated sleep. Pediatr Radiol. 2014;44(1):64-72.

42. Ashley WW Jr, McKinstry RC, Leonard JR, et al. Use of rapid-sequence magnetic resonance imaging for evaluation of hydrocephalus in children. J Neurosurg. 2005;103(2) (suppl):124-130.

\section{Disclosures}

Dr. Patel reports being a consultant for Stryker.

\section{Author Contributions}

Conception and design: Patel, Commean, Goyal, An. Acquisition of data: Eldeniz, Jammalamadaka, Goyal, Smyth. Analysis and interpretation of data: Patel, Eldeniz, Skolnick, Commean, An. Drafting the article: Patel, Skolnick, An. Critically revising the article: Patel, Smyth, An. Reviewed submitted version of manuscript: Patel, Commean, Smyth, An. Approved the final version of the manuscript on behalf of all authors: Patel. Statistical analysis: Skolnick. Study supervision: Patel.

\section{Supplemental Information \\ Online-Only Content}

Supplemental material is available with the online version of the article.

Supplemental Data. https://thejns.org/doi/suppl/10.3171/2020. 4.PEDS20131.

\section{Correspondence}

Kamlesh B. Patel: Washington University in St. Louis, MO. kamlesh.patel@wustl.edu. 